Supporting Information

\title{
Enzymatic Cleaning Mitigates Polysaccharide-Induced Refouling of RO Membrane: Evidence from Foulant Layer Structure and Microbial Dynamics
}

\author{
Yufang Li ${ }^{\text {a }}$, Han Wang ${ }^{\text {a }}$, Shu Wang ${ }^{\text {a }}$, Kang Xiao ${ }^{\text {b,c,*, Xia Huang a,c,* }}$ \\ ${ }^{\text {a }}$ State Key Joint Laboratory of Environment Simulation and Pollution Control, School of \\ Environment, Tsinghua University, Beijing 100084, China \\ ${ }^{\mathrm{b}}$ College of Resources and Environment, University of Chinese Academy of Sciences, Beijing, \\ 100049 China \\ ${ }^{\mathrm{c}}$ Research and Application Center for Membrane Technology, School of Environment, Tsinghua \\ University, Beijing 100084, China \\ *E-mail: kxiao@ucas.ac.cn (K.Xiao), xhuang@tsinghua.edu.cn (X. Huang)
}

Number of Pages: 16

Number of Figures: 5

Number of Tables: 9 


\section{Details of characterization method}

Part I. Foulant extraction analysis.

$\underline{\text { Page S3 }}$

Part II. Microbial analysis.

$\underline{\text { Page S3-S4 }}$

\section{Summary of figures and tables}

Figure S1. Brief illustration of the structure of cross-flow setup.

$\underline{\text { Page S5 }}$

Figure S2. Scanning electron microscope (SEM) images of virgin membrane (Virgin) and samples after different cleaning treatment.

$\underline{\text { Page S5 }}$

Figure S3. ATR-FTIR spectra of virgin and cleaned membranes.

$\underline{\text { Page S6 }}$

Figure S4. Characteristics of fluorescent organics, including 3D-EEM spectra of the feed and extractions of samples after cleaning and the corresponding FQ spectra.

$\underline{\text { Page S7 }}$

Figure S5. Illustration of the Z-stack scan process applied in this article.

Page S8

Table S1. Water quality of the coal chemical wastewater used as the feed.

$\underline{\text { Page S9 }}$

Table S2. Data-fitting result of adapted cross-flow filtration model.

$\underline{\text { Page S9 }}$

Table S3. Contact angle and desalination rate of membranes after long time treatment of different reagents.

$\underline{\text { Page S10 }}$

Table S4. Correlations between absorbance of characteristic peaks and cleaning efficiency $(\eta)$, surface roughness $\left(R_{\text {rms }}\right.$ value) and refouling constant $k$.

$\underline{\text { Page S10 }}$

Table S5. Intensity of representative peaks, and fluorescence indicator values of extracted organic matters on membranes.

$\underline{\text { Page S11 }}$

Table S6. Adenosine triphosphate (ATP) content and $\alpha$-diversity of microbial communities in residual biofilm on cleaned samples.

$\underline{\text { Page S11 }}$

Table S7. Topological properties of microbial communities.

$\underline{\text { Page S12 }}$

Table S8. Centrality indexes of key taxa in communities.

Page S13-14

Table S9. Result of market research of mainstream membrane cleaner products.

$\underline{\text { Page S15-16 }}$ 


\section{Detailed description of characterization methods}

Part I. Foulant extraction analysis

a. Total organic carbon: measured by TOC-Lcph analyzer (Shimadzu, Japan). After catalytic oxidation combustion at $680{ }^{\circ} \mathrm{C}$, subtraction method was used, i.e. total organic carbon $(\mathrm{TOC})=$ total carbon (TC) - total inorganic carbon (TIC).

b. Polysaccharides: measured by phenol sulfuric acid method. Samples was mixed with 5\% phenol and $98 \%$ sulfuric acid, with volume ratio of 1:1:3. After color reaction (30 min, room temperature), absorbance at $490 \mathrm{~nm}$ was determined ${ }^{1}$.

c. Protein: determined by BCA protein assay kit (Beyotime Biotechnology, China). According to the kit protocol, the mixture of $20 \mu \mathrm{L}$ sample and $200 \mu \mathrm{L}$ working solution was incubated for 30 min under $60{ }^{\circ} \mathrm{C}$ for sufficient color reaction. After that, the absorbance at $562 \mathrm{~nm}$ was measured.

d. Humic-like substances: measured by Lowry method. Absorbance at $750 \mathrm{~nm}$ was determined after samples reacted with Lowry I, Lowry II and Folin reagent, according to protocol described by Shen, et al. ${ }^{2}$.

e. Fluorescent matters: learned by three-dimensional excitation emission matrix (3D-EEM) on spectrophotometer Model F-7000 (Hitachi, Japan). Spectra within excitation of 200-400 nm and emission of 250-500 $\mathrm{nm}$ (5 $\mathrm{nm}$ stepwise) was collected. The obtained data were further processed after subtracting water background and eliminating Rayleigh and Raman scatterings ${ }^{3}$.

Part II. Microbial analysis

a. ATP content: determined by Ultrasnap ${ }^{\mathrm{TM}}$ (Hygiena, America), a kind of integrated surface sampling swab. The fluorescence of swab was immediately quantified by SystemSURE II Pi-102 (Hygiena, America), a kind of portable ATP fluorometer.

b. Distribution of biomass: samples was stained by $20 \mu \mathrm{M} \mathrm{SYTO}^{\mathrm{TM}} 63$ (Thermo Fisher Scientific, America), $0.2 \mathrm{~g} / \mathrm{L}$ Concanavalin A (Sigma, USA) and Calcofluor White (Sigma-Aldrich, USA) in sequence for the observation of nucleic acids, $\alpha$-pyranosyl sugar and $\beta$-D-glucopyranose polysaccharides, respectively ${ }^{4}$. The stained samples were observed by confocal laser scanning microscope (Zeiss LSM780, Germany), with emission signals on channel 650-690 nm, 550-600 $\mathrm{nm}$ and 420-470 nm collected. Z-stack scanning function (illustrated in Fig. S5) was also applied 
to obtain stereoscopic description of the matrix. Average fluorescence intensity (Avg. Inten.) and average density (Avg. den.) were represented by the mean gray value and percentage of biomass volume in the obtained 3D-matrix after 8-bit binary processing, respectively.

c. 16S rDNA sequencing: high-throughput sequencing of $16 \mathrm{~S}$ bacterial V3 and V4 regions was done on HiSeq-PE250 platform. From DNA extraction to sequencing, all process was implemented by Guangdong Magigene Biotechnology Co., Ltd. The sequencing reads were deposited on NCBI database under BioProject PRJNA649103.

\section{References}

(1) DuBois, M.; Gilles, K. A.; Hamilton, J. K.; Rebers, P. A.; Smith, F., Colorimetric Method for Determination of Sugars and Related Substances. Analytical Chemistry 1956, 28, (3), 350-356.

(2) Shen, Y. X.; Xiao, K.; Liang, P.; Ma, Y. W.; Huang, X., Improvement on the modified Lowry method against interference of divalent cations in soluble protein measurement. Applied Microbiology \& Biotechnology 2013, 97, (9), 4167-4178.

(3) Li, Y.; Li, M.; Xiao, K.; Huang, X., Reverse osmosis membrane autopsy in coal chemical wastewater treatment: Evidences of spatially heterogeneous fouling and organic-inorganic synergistic effect. Journal of Cleaner Production 2020, 246, 118964.

(4) Chen, M.-Y.; Duu-Jong, L.; Yang, Z.; Peng, X. F.; Lai, J. Y., Fluorecent staining for study of extracellular polymeric substances in membrane biofouling layers. Environmental Science \& Technology 2006, 40, (21), 6642-6. 


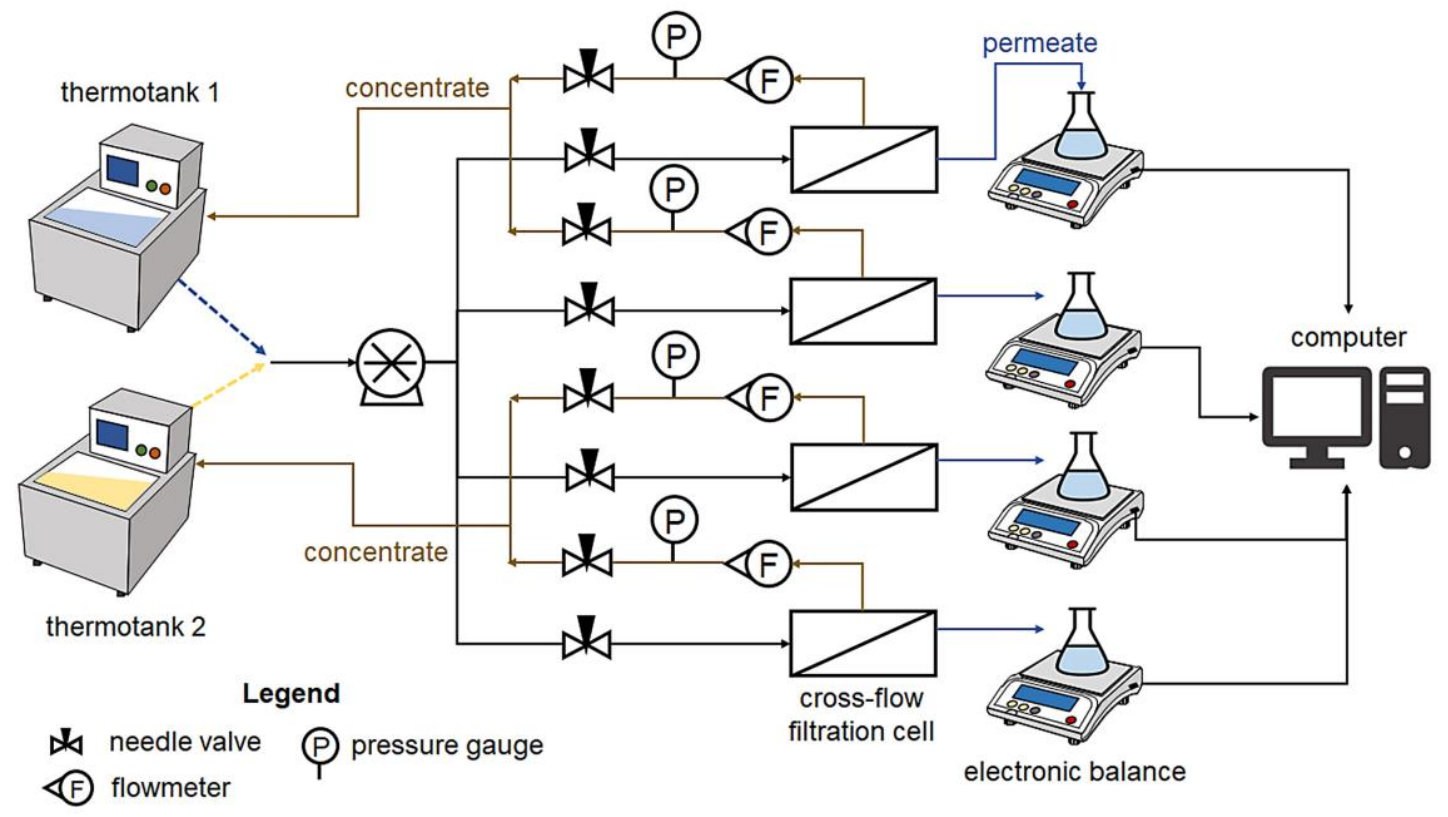

Figure S1. Brief illustration of the structure of cross-flow setup used for cleaning-in-place tests. Two thermotanks could be filled with either practical coal chemical wastewater (in fouling model) or cleaning reagents (for cleaning model). Through switching tanks, the switching between CIP mode and fouling mode was realized.

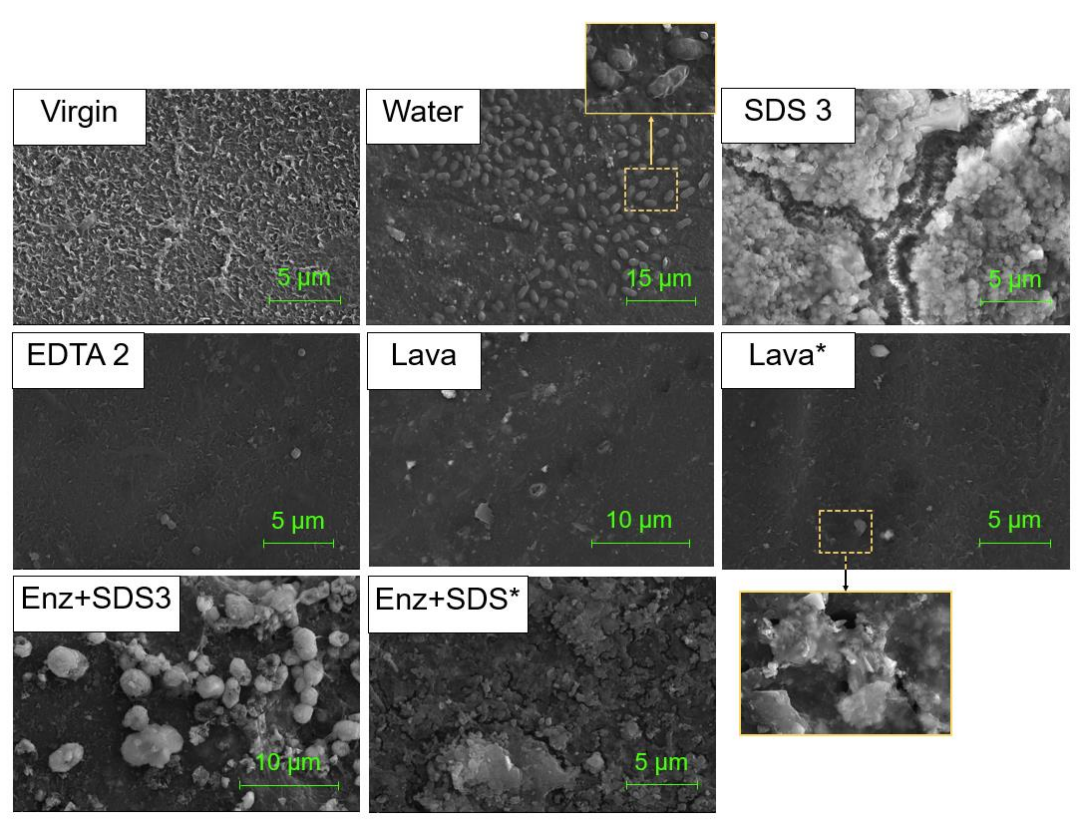

Figure S2. Scanning electron microscope (SEM) images of virgin membrane (Virgin) and samples after different CIP process. To emphasize the existence of microbial cells and gel-like matter, 12000times-magnified micro fields of Water sample and Lava* sample were also illustrated. Enz+SDS* and Lava* represented specimens sampled at point 3 (labeled in Figure 2) in long-term experiments. 

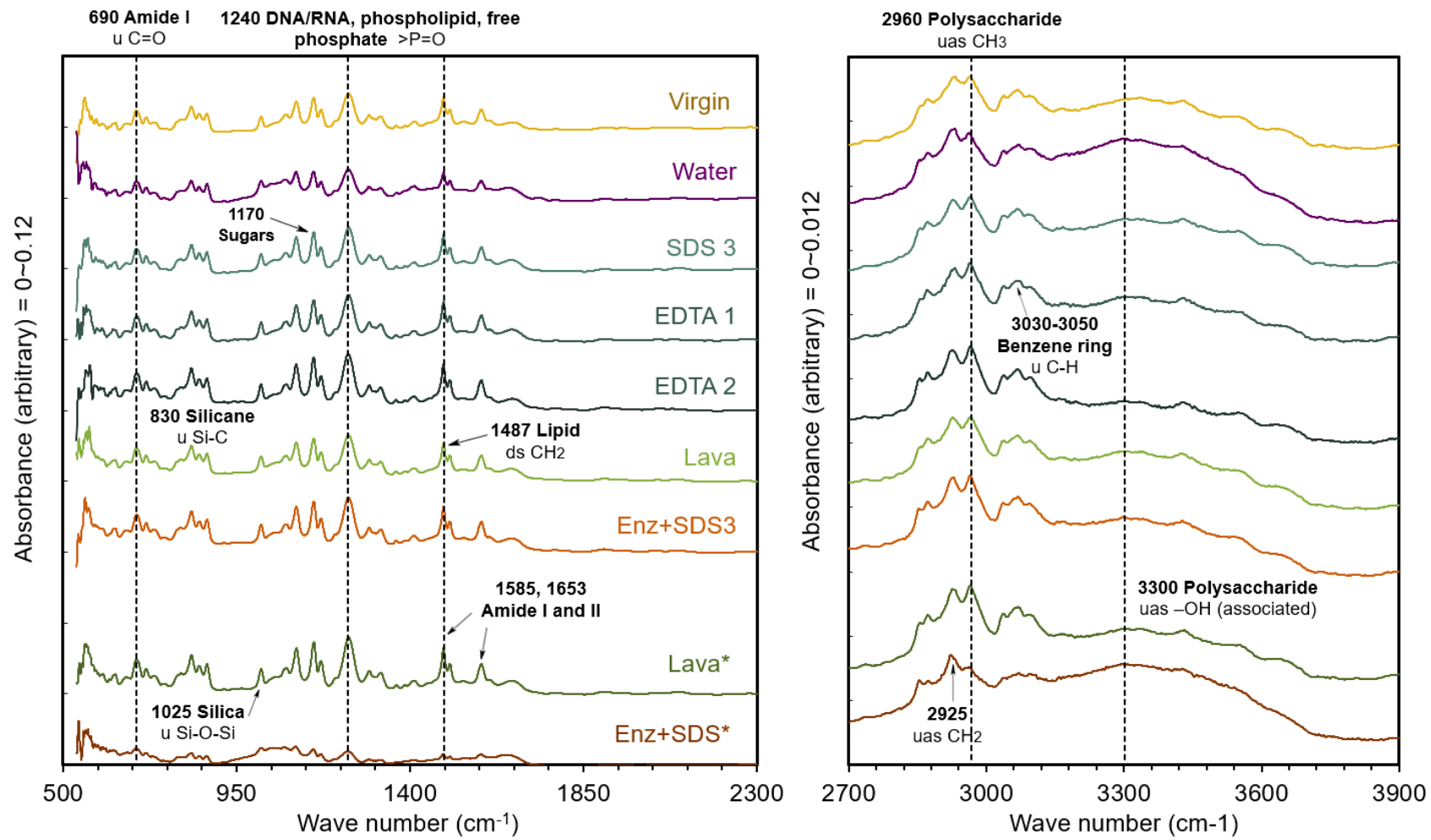

Figure S3. Attenuated total reflection Fourier transformed infrared (ATR-FTIR) spectra of the membrane surfaces after different cleaning treatments. To fully recognize the signal of foulants from the material of selective layer, i.e. polyamide, several initial characteristic peaks of polyamide with strong response are listed as below (take PA66 as example):

\begin{tabular}{|c|c|c|c|c|c|}
\hline $\begin{array}{c}\text { Wave number } \\
\qquad\left(\mathrm{cm}^{-1}\right)\end{array}$ & Bond & Category & $\begin{array}{c}\text { Wave number } \\
\quad\left(\mathrm{cm}^{-1}\right)\end{array}$ & Bond & Category \\
\hline 1276 & -CONH- & \multirow{3}{*}{$\begin{array}{l}\text { Bending } \\
\text { vibration }\end{array}$} & 2858 & $-\mathrm{CH}_{2-}$ & Symmetric stretching \\
\hline 1473 & $-\mathrm{CH}_{2-}$ & & 2934 & $-\mathrm{CH}_{2}-$ & $\begin{array}{l}\text { Asymmetric } \\
\text { stretching }\end{array}$ \\
\hline 1540 & -CO-NH- & & 3299 & $-\mathrm{NH}$ & Stretching vibration \\
\hline
\end{tabular}


(A) Fluorescence spectra of residual foulants and the feed
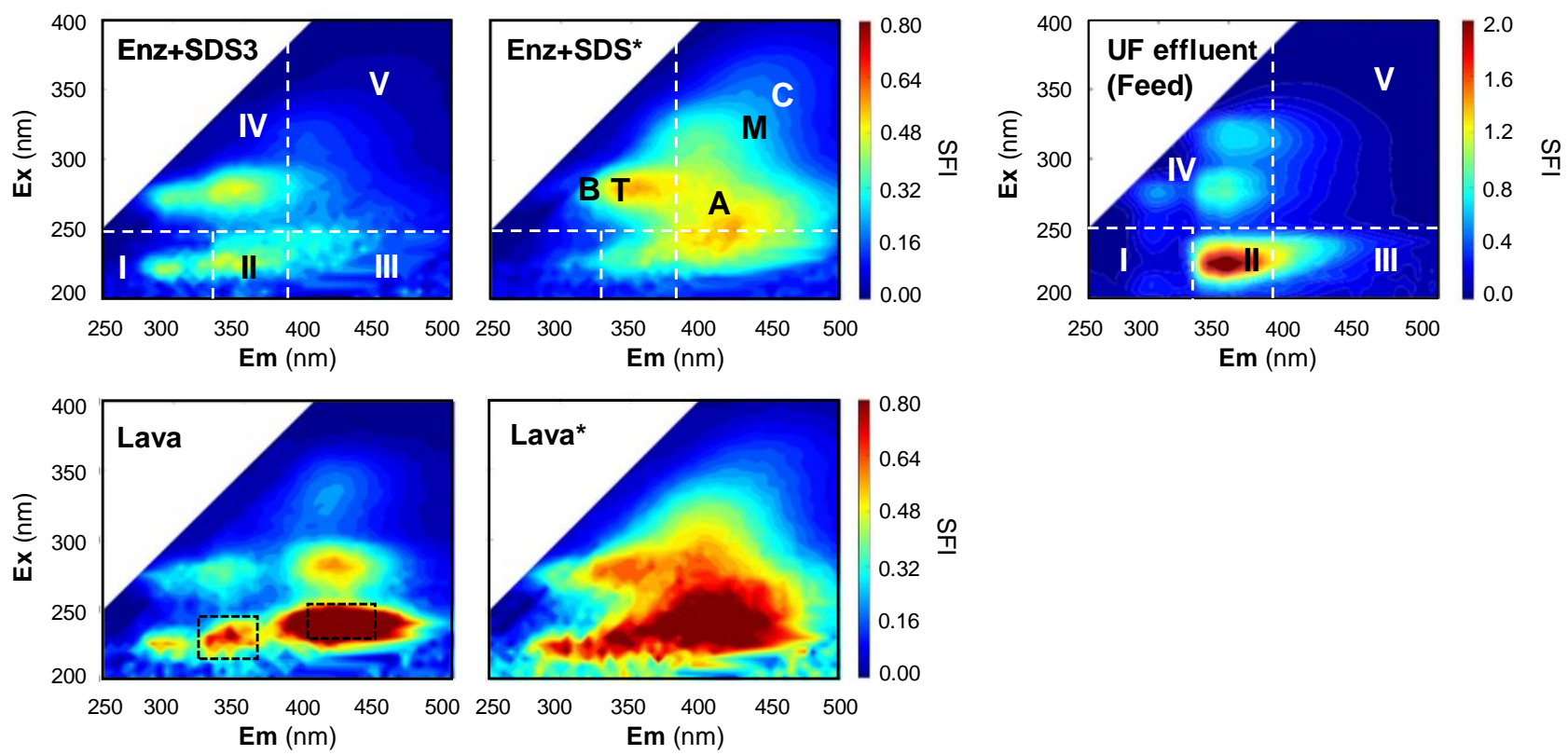

(B) Fluorescence quotient (FQ) spectra

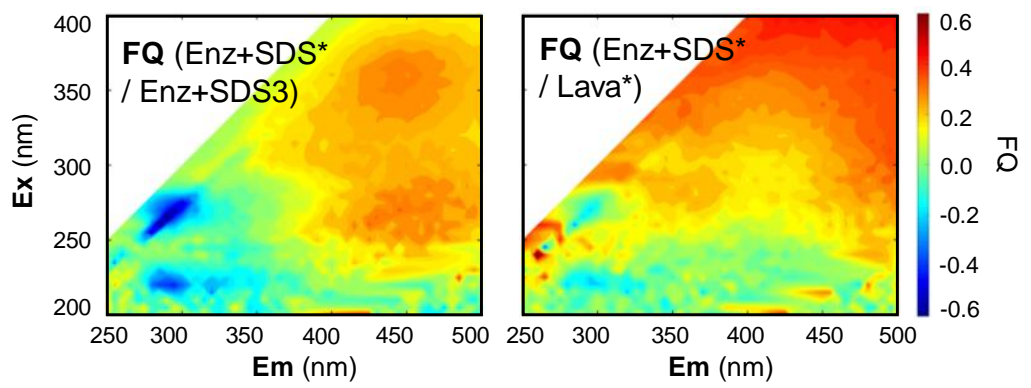

Figure S4. Characteristics of fluorescent organics. (A) 3D-EEM spectra of extracted residual foulants on cleaned membranes and the feed (i.e. ultrafiltration (UF) effluent of coal chemical wastewater), with the division of region I-V and positions of peak A, B, T, M, C labeled. Areas highlighted by black dashed line were peaks interfered by metallic ions-chelators interaction. (B) Fluorescence quotient (FQ) spectra, which reflected the long-term enzymatic selective effect on residual foulants (FQ (Enz+SDS*/ Enz+SDS3)) and the difference of foulants between enzymatic and harsh cleaning group (FQ (Enz+SDS*/ Lava*)). In both (A) and (B), Enz+SDS* and Lava* represented long-term specimens sampled after the third cleaning process. 
(A) Diagram of Z-stack scan

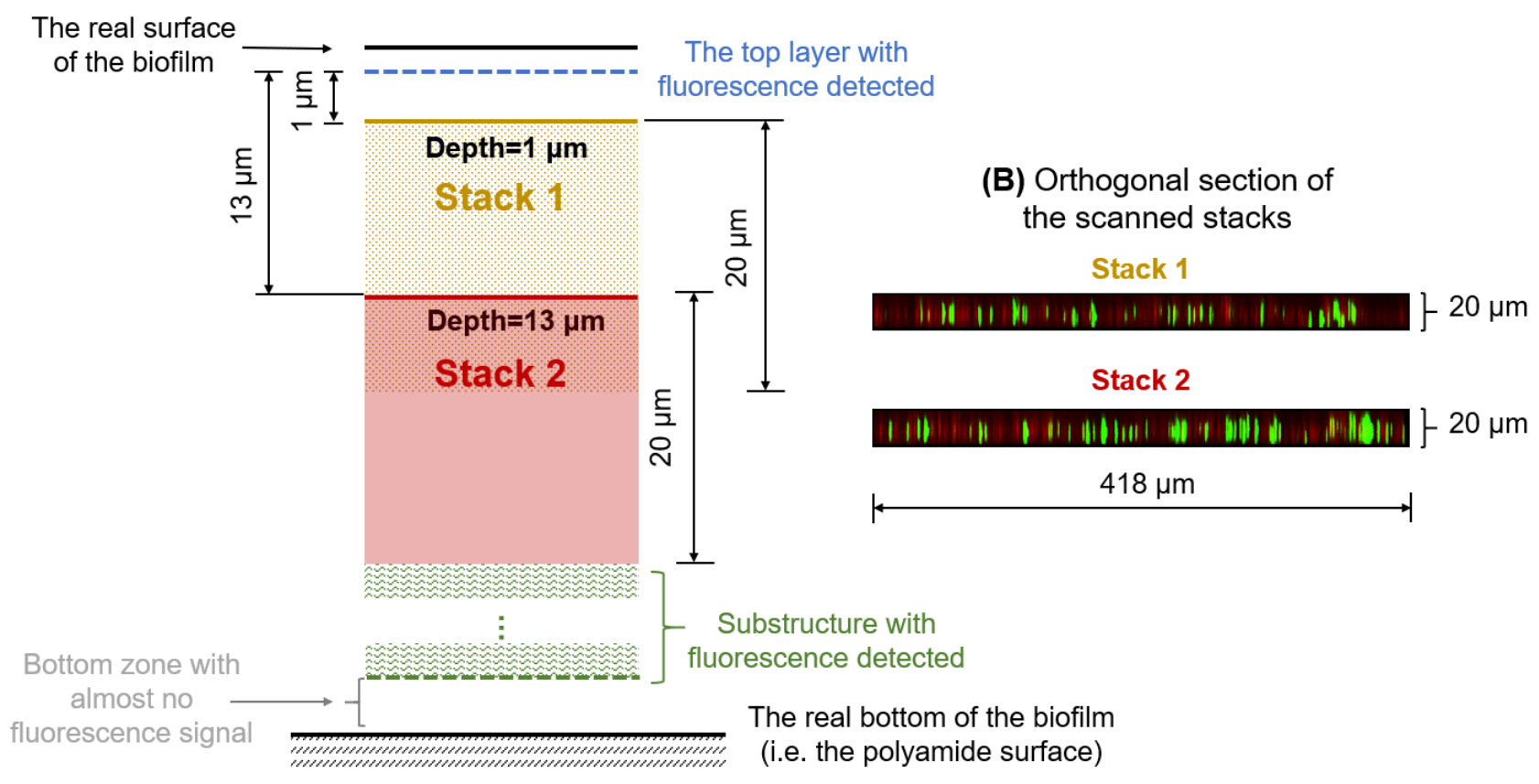

Figure S5. Illustration of the Z-stack scan process applied in this article. (A) Diagram of Z-stack scan, with clear clarification of the depth and thickness information of stack 1 and stack 2 . The observed thickness of fouling layer was defined as the distance between the blue and green dashed line. (B) Size of the orthogonal section of the scanned stacks. 
Table S1. Water quality of the coal chemical wastewater used as the feed.

\begin{tabular}{cccccccccc}
\hline $\mathrm{pH}$ & $\mathrm{SDI}^{a}$ & $\begin{array}{c}\text { Conductivity } \\
(\mu \mathrm{S} / \mathrm{cm})\end{array}$ & $\begin{array}{c}\mathrm{COD}^{a} \\
(\mathrm{mg} / \mathrm{L})\end{array}$ & $\begin{array}{c}\text { Hardness }^{b} \\
(\mathrm{mg} / \mathrm{L})\end{array}$ & $\begin{array}{c}\mathrm{TN} \\
(\mathrm{mg} / \mathrm{L})\end{array}$ & $\begin{array}{c}\mathrm{TP} \\
(\mathrm{mg} / \mathrm{L})\end{array}$ & $\begin{array}{c}\text { Proteins } \\
(\mathrm{mg} / \mathrm{L})\end{array}$ & $\begin{array}{c}\text { Polysaccharides } \\
(\mathrm{mg} / \mathrm{L})\end{array}$ & $\begin{array}{c}\text { Humics } \\
(\mathrm{mg} / \mathrm{L})\end{array}$ \\
\hline $8.0-$ & $<2.6$ & \multirow{2}{*}{$1940-2200$} & $\begin{array}{c}100- \\
120\end{array}$ & $<65$ & $<20$ & $<0.63$ & $\begin{array}{c}29.4- \\
33.2\end{array}$ & $22.0-25.7$ & $\begin{array}{c}35.2- \\
39.7\end{array}$ \\
\hline
\end{tabular}

${ }^{a}$ SDI, COD, TN, NH4+-N and TP stand for silting density index, chemical oxygen demands, total nitrogen, ammonia nitrogen and total phosphorus, respectively.

${ }^{b}$ Hardness was expressed as $\mathrm{CaO}$ equivalent

Table S2. Parameter values after fitting the flux data of long-term CIP tests in fouling model $\mathrm{d} J / \mathrm{d} t=$ $-k \cdot\left(J-J^{*}\right) \cdot J^{2-n}$. Among, $J$ and $J^{*}$ represented the membrane flux and steady flux (unit: LMH), respectively, while $t$ represented the corresponding fouling duration (unit: hour). Value of $\mathrm{R}^{2}$ was used to characterize the fitting accuracy, while constant $k$ was used to reflect re-fouling rate. The obtained $k$ in complete blocking model, intermediate blocking model, cake filtration model (or gel layer formation) and standard blocking model was represented as $k_{\mathrm{c}}, k_{\mathrm{i}}, k_{\mathrm{gl}}$ and $k_{\mathrm{s}}$, respectively.

\begin{tabular}{|c|c|c|c|c|c|c|c|}
\hline & \multirow{2}{*}{\multicolumn{2}{|c|}{ Model parameter }} & \multicolumn{2}{|c|}{ Cycle 1} & \multicolumn{2}{|c|}{ Cycle 2} & \multirow{2}{*}{ Cycle 3} \\
\hline & & & Parallel 1 & Parallel 2 & Parallel 1 & Parallel 2 & \\
\hline \multirow{8}{*}{ Enz+SDS } & \multirow{4}{*}{$\begin{array}{c}\text { Complete } \\
\text { blocking } \\
\text { Intermediate } \\
\text { blocking }\end{array}$} & $k_{\mathrm{c}}$ & 0.0010 & 0.0019 & 0.0067 & 0.0056 & 0.0106 \\
\hline & & $\mathrm{R}^{2}$ & 0.0001 & 0.0004 & 0.0100 & 0.0117 & 0.3104 \\
\hline & & $k_{\mathrm{i}}$ & 0.0008 & 0.0014 & 0.0003 & 0.0142 & 0.0122 \\
\hline & & $\mathrm{R}^{2}$ & $<0.0001$ & 0.0001 & $<0.0001$ & 0.0390 & 0.2343 \\
\hline & \multirow{2}{*}{ Cake filtration } & $k_{\mathrm{gl}}$ & 0.0040 & 0.00640 & 0.0143 & 0.0290 & 0.0127 \\
\hline & & $\mathrm{R}^{2}$ & 0.0012 & 0.0020 & 0.0093 & 0.0802 & 0.1461 \\
\hline & \multirow{2}{*}{$\begin{array}{l}\text { Standard } \\
\text { blocking }\end{array}$} & $\boldsymbol{k}_{\mathrm{s}}$ & 0.0019 & 0.0013 & 0.0018 & 0.0020 & 0.0018 \\
\hline & & $\mathbf{R}^{2}$ & 0.7336 & 0.6878 & 0.7215 & 0.8618 & 0.9530 \\
\hline \multirow{8}{*}{ Lava } & Complete & $k_{\mathrm{c}}$ & 0.0507 & 0.0429 & 0.0405 & 0.0209 & 0.0546 \\
\hline & blocking & $\mathrm{R}^{2}$ & 0.1867 & 0.1421 & 0.2426 & 0.0811 & 0.6684 \\
\hline & Intermediate & $k_{\mathrm{i}}$ & 0.0063 & 0.0456 & 0.0377 & 0.0166 & 0.0927 \\
\hline & blocking & $\mathrm{R}^{2}$ & $<0.0001$ & 0.0701 & 0.0040 & 0.0194 & 0.757 \\
\hline & \multirow{2}{*}{ Cake filtration } & $k_{\mathrm{gl}}$ & 0.0824 & 0.0403 & 0.0741 & 0.0028 & 0.1575 \\
\hline & & $\mathrm{R}^{2}$ & 0.0014 & 0.0210 & 0.0313 & 0.0002 & 0.7656 \\
\hline & \multirow{2}{*}{$\begin{array}{l}\text { Standard } \\
\text { blocking }\end{array}$} & $\boldsymbol{k}_{\mathrm{s}}$ & 0.0025 & 0.0021 & 0.0023 & 0.0021 & 0.0012 \\
\hline & & $\mathbf{R}^{2}$ & 0.7774 & 0.7189 & 0.7406 & 0.8025 & 0.5728 \\
\hline
\end{tabular}


Table S3. Properties variation after treatment of different reagents. Enz, Ctrl S, Lava and Pure water stands for the solution of the mixture of $250 \mathrm{ppm}$ lipase and $250 \mathrm{ppm}$ protease ( $\mathrm{pH}=9), 50 \mathrm{mg} / \mathrm{L}$ SDS $(\mathrm{pH}=9), 2 \%$ Lavasol $7(\mathrm{pH}=12.45)$ and ultrapure water, respectively. (1) Contact angle of the membranes after soaking for 2 weeks and rinsing to remove residual reagents. (2) After soaking for 2 hours: desalination rates of the membrane were measured on the cross-flow setup, with 2000 ppm $\mathrm{NaCl}$ as the feed.

\begin{tabular}{ccccc}
\hline & Enz & SDS 3 & Lava & Pure water \\
\hline Contact angle $\left(^{\circ}\right)$ & $73.02 \pm 4.52$ & $78.67 \pm 3.18$ & $52.80 \pm 3.67$ & $85.93 \pm 1.22$ \\
Desalination rate $(\%)$ & $97.3 \pm 0.5$ & $97.6 \pm 0.2$ & $92.2 \pm 0.8$ & $97.8 \pm 0.4$ \\
\hline
\end{tabular}

Table S4. Correlations between absorbance of characteristic peaks and surface roughness $\left(R_{\mathrm{rms}}\right.$ value $)$, cleaning efficiency $(\eta)$, as well as the value of $k_{s}$ derived in Table S2. In each cell of the table, the number above was Spearman correlation coefficient, while number beneath was the corresponding significance, i.e. $p$ value. Only correlations with relatively high significance $(p<0.2)$ were listed in the table.

\begin{tabular}{c|ccccc|cccc}
\hline $\begin{array}{c}\text { Wave number } \\
\left(\mathrm{cm}^{-1}\right)\end{array}$ & \multicolumn{5}{|c|}{ Fingerprint zone } & \multicolumn{4}{c}{ Functional group zone } \\
\cline { 2 - 10 } & 690 & 830 & 850 & 1013 & 1170 & 2960 & 3070 & 3300 & 3430 \\
\hline $\begin{array}{c}\text { Representated } \\
\text { organics }\end{array}$ & Proteins & Silica & & & $\begin{array}{c}\text { Poly- } \\
\text { saccharides }\end{array}$ & $\begin{array}{c}\text { Broad band of hydoxyls, probably } \\
\text { related to humics and polysaccharides }\end{array}$ \\
$R_{\text {rms }}$ & -0.486 & -0.490 & -0.486 & -0.402 & -0.402 & & & & \\
& $(0.078)$ & $(0.075)$ & $(0.078)$ & $(0.154)$ & $(0.154)$ & & & & \\
$k_{s}$ & 0.309 & & & & 0.309 & 0.309 & 0.265 & 0.315 & 0.265 \\
& $(0.125)$ & & & & $(0.082)$ & $(0.125)$ & $(0.188)$ & $(0.107)$ & $(0.107)$ \\
$\eta$ & & & & & & -0.380 & -0.429 & -0.385 & -0.385 \\
& & & & & & $(0.180)$ & $(0.126)$ & $(0.175)$ & $(0.175)$ \\
\hline
\end{tabular}


Table S5. Intensity of representative peaks, and fluorescence indicator values of extracted organic matters on membranes. Peak T, B, A, M, C represented tryptophan-like, tyrosine-like, humic-like, marine humic-like and older humic-like substances, respectively. Enz+SDS* and Lava* represented specimens sampled at point 3 (labeled in Figure 2) in long-term experiments.

\begin{tabular}{cccccc}
\hline & & Enz+SDS3 & Enz+SDS* & Lava & Lava* \\
\hline \multirow{3}{*}{ Specific Peak } & $\mathrm{A}$ & 548.5 & 946.1 & 849.1 & 742.7 \\
intensity & $\mathrm{C}$ & 72.9 & 360.8 & 77.3 & 135.4 \\
(a. u.) & $\mathrm{M}$ & 188.7 & 715.7 & 143.9 & 302.3 \\
& $\mathrm{~B}$ & 609.9 & 576.4 & 415.5 & 469.2 \\
& $\mathrm{~T}$ & 530.4 & 911.2 & 288.8 & 408.8 \\
\hline \multirow{3}{*}{ Intensity ratio } & $\mathrm{BIX}$ & $1.31 \pm 0.12$ & $1.08 \pm 0.07$ & $1.15 \pm 0.08$ & $1.07 \pm 0.10$ \\
& $f_{450} / f_{500}$ & $1.69 \pm 0.18$ & $1.72 \pm 0.10$ & $2.33 \pm 0.04$ & $1.71 \pm 0.04$ \\
& $\mathrm{HP}$ & $1.41 \pm 0.12$ & $2.22 \pm 0.16$ & $2.45 \pm 0.14$ & $1.97 \pm 0.12$ \\
\hline
\end{tabular}

Table S6. Adenosine triphosphate (ATP) content and $\alpha$-diversity of microbial communities of residual biofilm on Enz+SDS* and Lava* specimens sampled at point 2 and 3 (labeled in Figure 2), respectively.

\begin{tabular}{|c|c|c|c|c|c|}
\hline & & \multicolumn{2}{|c|}{$\begin{array}{c}\text { Enzymatic cleaning } \\
(\text { Enz+SDS*) }\end{array}$} & \multicolumn{2}{|c|}{$\begin{array}{l}\text { Harsh alkaline cleaning } \\
\text { (Lava*) }\end{array}$} \\
\hline & & Sample 2 & Sample 3 & Sample 2 & Sample 3 \\
\hline ATP cc & ent (RLUs) & $2643 \pm 232$ & $3054 \pm 311$ & $229 \pm 18$ & $69 \pm 9$ \\
\hline \multirow{2}{*}{$\alpha$-diversity } & Chaol index & $383.0 \pm 29.5$ & $459.1 \pm 34.3$ & $386.8 \pm 17.1$ & $539.6 \pm 27.8$ \\
\hline & Shannon index & $4.6 \pm 0.3$ & $5.9 \pm 0.5$ & $5.4 \pm 0.2$ & $6.0 \pm 0.3$ \\
\hline
\end{tabular}


Table S7. Topological properties of microbial communities.

\begin{tabular}{|c|c|c|}
\hline Network indexes & Enzymatic cleaning & Harsh cleaning \\
\hline Similarity threshold (St) & 0.92 & 0.90 \\
\hline Total nodes (n) & 195 & 133 \\
\hline Total links & 394 & 129 \\
\hline $\mathrm{R}^{2}$ of power-law & 0.843 & 0.878 \\
\hline Efficiency & 0.918 & 0.969 \\
\hline Average degree (avgK) & 4.041 & 2.942 \\
\hline $\begin{array}{l}\text { Average clustering } \\
\text { coefficient (avgCC) }\end{array}$ & 0.394 & 0.251 \\
\hline Average path distance (GD) & 4.169 & 6.203 \\
\hline Geodesic efficiency (E) & 0.375 & 0.235 \\
\hline Modularity & 0.789 & 0.803 \\
\hline Module & 27 & 34 \\
\hline Density (D) & 0.021 & 0.015 \\
\hline Reciprocity & 1 & 1 \\
\hline Transitivity (Trans) & 0.594 & 0.386 \\
\hline Connectedness (Con) & 0.149 & 0.349 \\
\hline Maximal degree & 18 & 10 \\
\hline Nodes with max degree & $\begin{array}{l}\text { OTU } 26 \text { (f_Moraxellaceae, } \\
\text { g_Alkanindiges) } \\
\text { OTU } 38 \text { (f_Moraxellaceae, } \\
\text { g_Cavicella) } \\
\text { OTU } 67 \text { (f_Burkholderiaceae, } \\
\text { g_Rhizobacter) }\end{array}$ & $\begin{array}{l}\text { OTU } 45 \text { (f_ } \\
\text { Chitinophagaceae, } \\
\text { g_Ferruginibacter) } \\
\text { OTU } 31 \\
\text { (f_Xanthobacteraceae, } \\
\text { g_Xanthobacter) }\end{array}$ \\
\hline Centralization of degree (CD) & 0.073 & 0.038 \\
\hline Maximal betweenness & 639 & 2597 \\
\hline Nodes with max betweenness & $\begin{array}{c}\text { OTU } 145 \\
\text { (f_- Moraxellaceae, } \\
\text { g_Acinetobacter) }\end{array}$ & $\begin{array}{c}\text { OTU } 48 \\
\text { (f_Verrucomicrobiaceae, } \\
\text { g_Prosthecobacter) }\end{array}$ \\
\hline Centralization of betweenness (CB) & 0.032 & 0.136 \\
\hline Maximal stress centrality & 4028 & 17495 \\
\hline Nodes with max stress centrality & $\begin{array}{c}\text { OTU } 145 \\
\text { (f__oraxellaceae, } \\
\text { g_Acinetobacter) }\end{array}$ & $\begin{array}{c}\text { OTU } 57 \\
\text { (f_Micrococcaceae, } \\
\text { g_Paeniglutamicibacter) }\end{array}$ \\
\hline $\begin{array}{c}\text { Centralization of stress centrality } \\
\text { (CS) }\end{array}$ & 0.198 & 0.914 \\
\hline Maximal eigenvector centrality & 0.302 & 0.424 \\
\hline $\begin{array}{c}\text { Centralization of eigenvector } \\
\text { centrality (CE) }\end{array}$ & 0.279 & 0.406 \\
\hline
\end{tabular}


Table S8. Centrality indexes of key taxa in biofilm communities of harsh cleaning group (sub-table 1) and mild cleaning group (sub-table 2). OTU IDs were sorted by node degree. Node. betw., node. evcent. and clustering coeffi., represented the betweenness, eigenvector centrality and clustering coefficient of the nodes, respectively. Moreover, nodes belonging to $\alpha$ - and $\gamma$-proteobacteria were labeled in orange and blue, respectively.

\section{(1) Harsh cleaning group}

\begin{tabular}{|c|c|c|c|c|c|c|c|c|c|}
\hline OTU ID & $\begin{array}{c}\text { Topological } \\
\text { role }\end{array}$ & $\begin{array}{l}\text { Node. } \\
\text { degree }\end{array}$ & $\begin{array}{l}\text { Node. } \\
\text { betw. }\end{array}$ & $\begin{array}{l}\text { Node. } \\
\text { stress }\end{array}$ & $\begin{array}{l}\text { Node. } \\
\text { evcent. }\end{array}$ & $\begin{array}{c}\text { Clustering } \\
\text { coeffi. }\end{array}$ & $\mathrm{Zi}$ & $\mathrm{Pi}$ & Family \\
\hline 45 & Module hubs & 10 & 19.92 & 48 & 0.423 & 0.467 & 2.88 & 0.51 & Chitinophagaceae \\
\hline 31 & Module hubs & 10 & 311.73 & 2673 & 0.002 & 0.250 & 3.02 & 0.28 & Xanthomonadales \\
\hline 440 & Module hubs & 9 & 1527.39 & 9764 & $<0.001$ & 0.389 & 2.54 & 0.13 & Burkholderiaceae \\
\hline 177 & & 9 & 327.08 & 1570 & 0.356 & 0.417 & 1.72 & 0.00 & Parachlamydiaceae \\
\hline 174 & & 9 & 785.33 & 6015 & 0.392 & 0.500 & 1.34 & 0.20 & Hyphomicrobiaceae \\
\hline 48 & & 8 & 2597.04 & 15401 & 0.007 & 0.214 & 1.11 & 0.38 & Verrucomicrobiaceae \\
\hline 16 & & 8 & 1787.99 & 13341 & 0.019 & 0.214 & 1.52 & 0.47 & Rhodocyclaceae \\
\hline 20 & & 8 & 616.32 & 5458 & $<0.001$ & 0.500 & 1.55 & 0.00 & Burkholderiaceae \\
\hline 424 & & 8 & 606.49 & 5284 & $<0.001$ & 0.536 & 1.55 & 0.00 & Sphingomonadaceae \\
\hline 159 & & 7 & 1001.49 & 9577 & $<0.001$ & 0.500 & -0.19 & 0.32 & Flavobacteriaceae \\
\hline 71 & & 7 & 979.39 & 3754 & 0.016 & 0.190 & 1.52 & 0.45 & Caedibacteraceae \\
\hline 23 & & 7 & 440.56 & 2908 & 0.002 & 0.333 & 1.68 & 0.00 & Caulobacteraceae \\
\hline 36 & & 7 & 331.89 & 2211 & 0.002 & 0.143 & 1.67 & 0.00 & Sphingomonadaceae \\
\hline 92 & & 7 & 305.37 & 1573 & 0.003 & 0.190 & 1.67 & 0.00 & Beijerinckiaceae \\
\hline 6 & Connectors & 7 & 1147.98 & 5541 & 0.009 & 0.286 & -0.03 & 0.69 & Burkholderiaceae \\
\hline 27 & Connectors & 5 & 2103.48 & 14023 & 0.001 & 0.100 & 0.52 & 0.66 & Dehalococcoidaceae \\
\hline 57 & Connectors & 4 & 2516.86 & 17495 & $<0.001$ & 0.167 & -1.07 & 0.63 & Micrococcaceae \\
\hline
\end{tabular}


(2) Mild cleaning group

\begin{tabular}{|c|c|c|c|c|c|c|c|c|c|}
\hline $\begin{array}{l}\text { OTU } \\
\text { ID }\end{array}$ & $\begin{array}{c}\text { Topological } \\
\text { role }\end{array}$ & $\begin{array}{l}\text { Node. } \\
\text { degree }\end{array}$ & $\begin{array}{l}\text { Node. } \\
\text { betw. }\end{array}$ & $\begin{array}{l}\text { Node. } \\
\text { stress }\end{array}$ & $\begin{array}{l}\text { Node. } \\
\text { evcent }\end{array}$ & $\begin{array}{c}\text { Clustering } \\
\text { Coeffi. }\end{array}$ & $\mathrm{Zi}$ & $\mathrm{Pi}$ & Family \\
\hline 26 & & 18 & 96.16 & 1194 & 0.302 & 0.549 & 1.77 & 0.00 & Moraxellaceae \\
\hline 38 & & 18 & 83.98 & 976 & 0.299 & 0.542 & 1.77 & 0.00 & Moraxellaceae \\
\hline 114 & & 16 & 343.78 & 2270 & 0.277 & 0.592 & 1.18 & 0.12 & Microscillaceae \\
\hline 124 & & 15 & 68.03 & 840 & 0.253 & 0.581 & 1.17 & 0.00 & Verrucomicrobiaceae \\
\hline 45 & & 15 & 107.91 & 1248 & 0.245 & 0.562 & 1.02 & 0.00 & Chitinophagaceae \\
\hline 365 & & 13 & 153.21 & 1196 & 0.231 & 0.703 & 0.56 & 0.14 & Flavobacteriaceae \\
\hline 71 & & 12 & 9.10 & 97 & 0.227 & 0.788 & 0.56 & 0.00 & Caedibacteraceae \\
\hline 195 & Module hubs & 11 & 57.97 & 710 & 0.178 & 0.709 & 2.74 & 0.00 & Pseudomonadaceae \\
\hline 80 & & 11 & 36.66 & 410 & 0.183 & 0.636 & 0.36 & 0.00 & Sphingomonadaceae \\
\hline 166 & Module hubs & 10 & 162.89 & 452 & $<0.001$ & 0.333 & 3.35 & 0.00 & NS11-12 marine group \\
\hline 310 & Module hubs & 10 & 4.58 & 93 & 0.200 & 0.867 & 2.62 & 0.34 & Sphingomonadaceae \\
\hline 135 & Module hubs & 6 & 161.64 & 1255 & 0.002 & 0.333 & 2.97 & 0.15 & Rhodocyclaceae \\
\hline 96 & Module hubs & 6 & 239.00 & 1709 & $<0.001$ & 0.200 & 2.65 & 0.00 & Pseudomonadaceae \\
\hline 145 & Connectors & 5 & 639.00 & 4028 & 0.060 & 0.300 & -1.47 & 0.70 & Moraxellaceae \\
\hline 49 & Module hubs & 5 & 17.52 & 20 & $<0.001$ & 0.206 & 2.56 & 0.44 & Weeksellaceae \\
\hline 76 & Connectors & 3 & 158.77 & 988 & 0.0145 & 0.328 & -0.93 & 0.63 & Parachlamydiaceae \\
\hline 386 & Connectors & 2 & 308 & 2618 & $<0.001$ & 0.000 & -0.55 & 0.74 & Burkholderiaceae \\
\hline 275 & Connectors & 2 & 255 & 70 & $<0.001$ & 0.000 & -1.32 & 0.65 & Spirosomaceae \\
\hline
\end{tabular}


Table S9. Result of market research of mainstream membrane cleaner products.

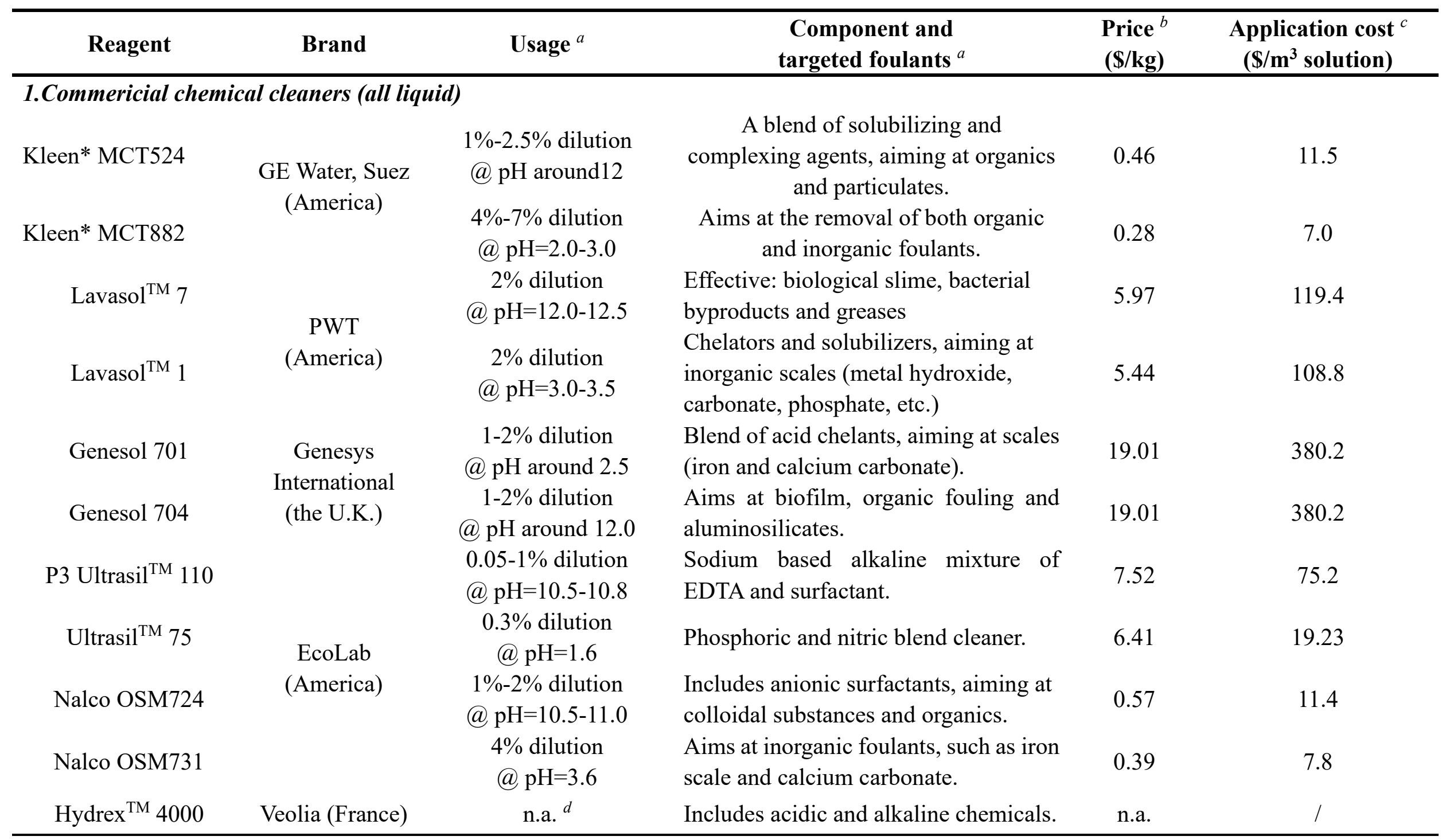

2. Pure chemical 
Sodium dodecyl sulfate (powder)

Macklin

Analytical grade

(purity: 92.5\%-99.5\%)

14.02

0.70

\section{Commercial Enzymatic reagents (all liquid)}

Progress Uno

Savinase Evity 16L

Lipex Evity 200L

Pectinex Ultra

Ultraflo Max

Mannaway 200L

Celluclean $5000 \mathrm{~L}$

Carezyme Premium $4500 \mathrm{~L}$

Ultrasil $^{\mathrm{TM}} 63$

Ultrasil $^{\mathrm{TM}} 67$

BIOREM ${ }^{\circledR}$ CIP
Medley ${ }^{\circledR}$ Advance

\section{Prefer neutral $\mathrm{pH}$}

Active among $\mathrm{pH}=7-11$

Active among $\mathrm{pH}=5-11$

Prefer $\mathrm{pH}=3.0-5.0$

Prefer $\mathrm{pH}=4.0-5.5$

Prefer $\mathrm{pH}=5.5-7.5$

Prefer $\mathrm{pH}=5.5-7.0$

Prefer $\mathrm{pH}$ around 4.5

Prefer $\mathrm{pH}$ around 5

$$
\text { n.a. }
$$

EcoLab

(America)

Realco
(Belgium)

$0.2 \%-0.5 \%$ dilution

(a) $\mathrm{pH}=9.75-10.5$

$1 \%$ dilution

Prefer $\mathrm{pH}=9-10$
Mainly composed of protease

Mainly alkaline protease.

A kind of lipase blend.

Pectinase and polygalacturonase.

Includes xylanase and $\beta$-dextranase.

Mannanase, aiming at adhesive gum.

Mainly composed of cellulase.

Subtilisin, $\alpha$-amylase and cellulase

Protease and anionic surfactants

Protease and nonionic surfactants

Enzymatic detergent, including

subtilisin, $\alpha$-amylase and cellulase, aiming at organic matrix of biofilms.
1.84

1.35

1.62

1.44

1.93

2.94

2.14

2.50

1.16

n.a.

15.55

l

n.a.

0.34

0.41
${ }^{a}$ Information is derived from product brochure, manual and introduction on websites.

${ }^{b}$ Price: product selling price offered by retailers.

${ }^{c}$ Application cost $=($ selling price $(\$ / \mathrm{kg}) /$ density $(\mathrm{kg} / \mathrm{L})) \times$ highest dilution level in usage $\times 1000\left(\mathrm{~m}^{3} / \mathrm{L}\right)$

${ }^{d}$ n.a.: information not available. 